\title{
SELENIUM IN CERTAIN METAMORPHIC ROCKS
}

\author{
TAPIO KolJONEN
}

Koljonen, Tapio 1973: Selenium in certain metamorphic rocks. Bull. Geol. Soc. Finland 45, 107-117.

The selenium contents of Precambrian metamorphic rocks from Finland were studied and tentative averages are presented. The pattern of the distribution of selenium in metamorphic rocks can be summarized as follows:

Selenium most probably does not enter into the structures of the common silicate minerals, but rather is incorporated in the accessory sulphide phase. The distribution of selenium in the rocks studied greatly resembles that distribution which is found in sediments. The highest contents are found in mica schists, phyllites and especially in sulphide bearing black schists. These are all metamorphosed clays possibly containing organic matter before metamorphism. In exogenic processes selenides are oxidised to elemental selenium which, being colloidal, is electrically charged and adsorbed by clay minerals.

Selenium contents are low in silicium rich rocks. In many gneisses and clastic quartzites the contents are less than $20 \mathrm{ppb}$, which is the lower limit of the analytical method used. Rocks which are derived of calcium and magnesium rich carbonate sediments are also nearly devoid of this element. The selenium that is found in the latter probably reflects the content of clay fraction and organic matter in the primary sediment.

Under metamorphism the rocks seem to loose a great part of that selenium which has originally been present in the unmetamorphosed sediment.

Tapio Koljonen, Department of Geology and Mineralogy, University of Helsinki, 00170 Helsinki 17, Finland.

\section{Introduction}

As a part of a study of the distribution and geochemical behaviour of selenium in the Finnish Precambrian, this paper deals with the highly metamorphic rocks.

Previous data for the contents of selenium in the metamorphic rocks of Finland are very scanty, the only work known to the author being that of Peltola (1960) which gives selenium contents in the black schist of the Outokumpu region.

The distribution of selenium in low metamorphic sedimentary rocks is quite well known especially in seleniferous areas were selenium is found in toxic concentrations (Beath and Gilbert 1936; Beath, Gilbert and Eppson 1937; Beath, Hagner and Gilbert 1946; Buryanova 1961; Coleman 1956; Coleman and Delevaux 1957; Davidson and Powers 1959; Krauskopf 1955; 
Moxon, Olson, Searight and Sandals 1938; Searight, Moxon, Hilmoe and Whitehead 1946; Trelease and Beath 1949; Williams and Byers 1935; and 1936).

The geochemical behaviour of selenium is dominated by its isomorphic substitution with sulphur in sulphides and consequently its geochemistry, to a high degree, resembles that of sulphur.

In the following the selenium contents of the various metamorphic rock groups are briefly reviewed.

\section{Gneisses}

On the basis of the data summarized in Table 1, Nos. 1-36, the selenium contents in gneissose rocks are diagrammatically presented in Fig. 1.

The selenium contents varies within relatively wide limits and depends largely on the amount of sulphide phase present. In many of the gneisses studied the selenium content was below the limit of detection of the method used. The low content in high siliceous gneisses parallels that of granites (Brunfelt and Steinness 1967; Koljonen 1971).

\section{Quartzites, meta-arkose and graywacke}

Table 1, Nos. 37-46, histogram in Fig. 1. The content of selenium is quite low. It is, however, interesting to note that selenium is found at all in this rock group. The selenium was probably adsorbed in clay minerals and partly retained during the metamorphism. Under mechanical and chemical weathering the selenium-bearing minerals (sulphides and dark minerals) are likely to be disintegrated. Clastic quartzites are therefore devoid of this element and selenium preferably occurs in sediments formed partly of siliceous gels. High selenium concentrations are known from Icelandic geyserites (Koljonen 1971).

Huhma (1970a, 63; and b, 24-26) suggested that some quartzites in the Outokumpu area are not sedimentary quartzites as proposed earlier. According to him no clastic textures can be observed in these rocks and the trace element pattern differs from that of sands.

Quartzites which are partly or wholly formed from $\mathrm{SiO}_{2}$-gels should contain more selenium than the clastic quartzites proper.

\section{Conglomerates}

Table 1, Nos. 67-70, histogram in Fig. 1.

The selenium content is transitional between those of the mica schists and sedimentary amphibolites usually constituting the matrix and, on the other hand, quartzites and magmatic rocks which form pebbles.

\section{Mica schists, phyllites and black schists}

Table 1, Nos. 47-60, histogram in Fig. 1. The selenium contents as illustrated in the histogram of Fig. 1, are high. Mica schists are metamorphic derivatives of clays in which selenium is adsorbed. The selenide ion is not easily oxidised to soluble selenite but to elemental selenium which is stable under the most exogenic conditions. As it is colloidal, it is easily adsorbed to clay minerals and other colloids (cf. Coleman and Dlevaux 1957; Fleming and Walsh 1957; Green 1959; Miller 1937; Miller and Brown 1938; Moxon, Olson, Searight and Sandals 1938; Searight, Moxon, Hilmoe and Whitehead 1946; Williams and Byers 1935; and 1936). Selenium participates in the biogeochemical processes and is enriched in black schists containing graphite and sulphides in abundance (cf. Beath and Gilbert 1936; Beath, Hagner and Gilbert 1946; Byers and Knight 1935; Byers, Williams and Lakin 1936; Chentzov 1963; Davidson and Gulbrandsen 1957; Fleming and Walsh 1957; Gibson and Selvig 1944; Keys and White 1956; Lakin, Williams and Byers 1938; Meixner 1954, 20; Miller 1937; Oksanen 1965, 
Robinson 1933). The varved schist, Ylöjärvi, Siivikkala! (Table 1, No. 48) contains $300 \mathrm{ppb}$ selenium, one of the highest Se concentration found in mica schists. The same rock contains the carbon sacks "Corycium enigmaticum» which, according to Sederholm $(1897,91)$ and Rankama (1948, 389-416; and 1950, 75) represent fossils, the carbon being of organic origin. This interpretation is in accordance with the high selenium content.

The high selenium contents found in black schists by the author are in accordance with those presented by Peltola (1960). The selenium content can vary in black schists within very wide limits according to their sulphide contents and the content of the original organic matter.

\section{Amphibolites}

The selenium contents are presented in Table 1, Nos. 61-66, and the histogram of Fig. 1. The contents range between those of mica schists and carbonate rocks.

\section{Carbonate rocks}

The selenium contents (Table 1, Nos. $71-75$, Fig. 1) are very low, this being in accordance with the chemistry of selenium. In basic surroundings it forms soluble salts - selenites which can easily migrate out of the system and may participate in the biocycle (Olson, Jornlin and Moxon 1942; Olson, Whitehead and Moxon 1942). The carbonatite of Silinjärvi, Finland, (Koljonen 1973) is probably of magmatic origin and is discussed in connection with the magmatic rocks (Puustinen 1968; and 1969).

\section{Miscellaneous schists and rocks}

Table 1, Nos. 76-80.

The selenium content is low. Due to the scarcity of available data the distribution will not be discussed in this connection. The ultrabasic rock No. 80 occurs in limestone and accordingly does not contain any selenium, an interesting observation since the sample abundantly contains biotite which in mica schists and especially in plutonic rocks often indicates high selenium contents (Koljonen 1971, 9).

\section{Skarn rocks}

Table 1, Nos. $81-82$.

This rock type also includes diopside gneiss No. 8. All the specimens show significantly low selenium contents. These rocks are probably formed in basic oxidising conditions.

The skarn rocks connected with sulphidic ores are excluded in this connection and discussed separately.

\section{Serpentine rocks}

Table 1. Nos. $83-86$.

The selenium contents are high in this rock group. In the Outokumpu region the serpentine rocks have been interpreted as products of hydrothermal metamorphism (Haapala 1936; Wiik 1953), apparently caused by selenium-rich emanations. Serpentine rocks are often connected with sulphidic ore mineralisations in this area and the high sulphur content in serpentine rocks is demostrated by the analyses given by Haapala (1936, 61-62) and Wiik (1953, 48-55).

\section{Metamorphic rocks of volcanogenic origin}

Table 1, Nos. 87-96. The selenium contents are lower than those commonly found in presentday volcanic rocks (cf. Bellini 1907; Brown 1916; Byers, William and Lakin 1936; Clarke 1924; Davidson and Powers 1959; Quercigh 1925; Zambonini and Coniglio 1925; Zies 1929, $26-27,40-41)$. In volcanogenic metamor- 
phic rocks the selenium contents have been appreciably decreased by exogenic processes (weathering and low temperature hydrothermal leaching) and by metamorphism. During weathering of low iron rocks with a high silicium content, the selenium begins to migrate and may, if the selenium content is sufficiently high, cause toxic diseases in animals (Trelease and Beath 1949).

\section{Dynamometamorphic rock}

The sample (Table 1, No. 97) is from s small, nearly black and about $30 \mathrm{~cm}$ broad vein in gneiss. Since the selenium content of the gneiss is very low, it is interesting to find some selenium in this glassy silica-rich vein. Selenium usually tends to follow iron in rocks. The phenomenon of the enrichment of selenium in vein rocks has been discussed by Koljonen (1971, 11-12). It seems obvious that selenium begins to migrate and tends to move along fissures and shear zones. The same phenomenon is seen in the mica schist No. 51, which displays well developed microfolds. In the rocks selenium is enriched in the small quartz vein. Peltola (1960, 73-74) observed an analogous phenomenon in black schist. He states: »The abundant occurrence of sulphide in the carbon-poor layers in black schist as conformable concentrations would seem to indicate their mobilization during the inital stage of deformation particularly in shear zones parallel to the bedding of black schist».

\section{Shock-metamorphic rocks}

The petrological study of these rocks was made by M. Lehtinen, who kindly placed several samples of the Lappajärvi shock-metamorphic rocks at the author's disposal (Table 1, Nos. 98-101). The selenium content is unusually high compared with other rocks of high $\mathrm{SiO}_{2}$
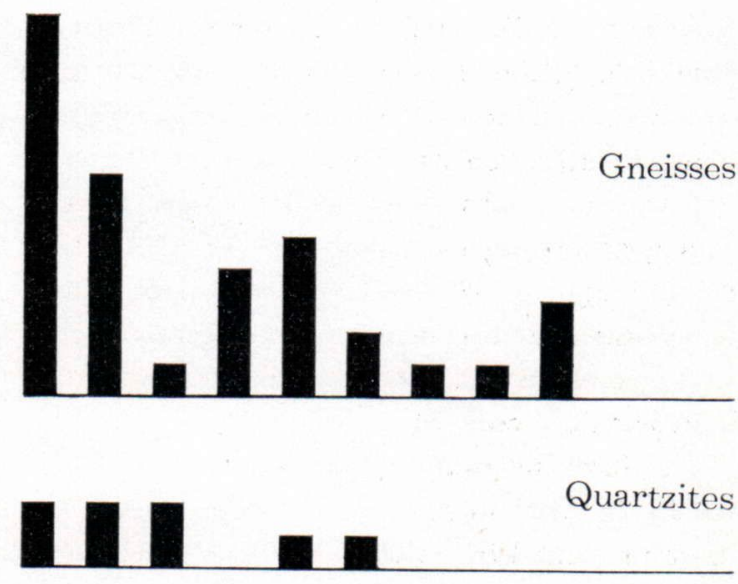

Conglomerates

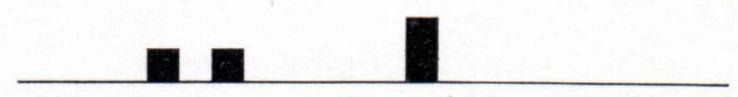

Mica schists Black schists
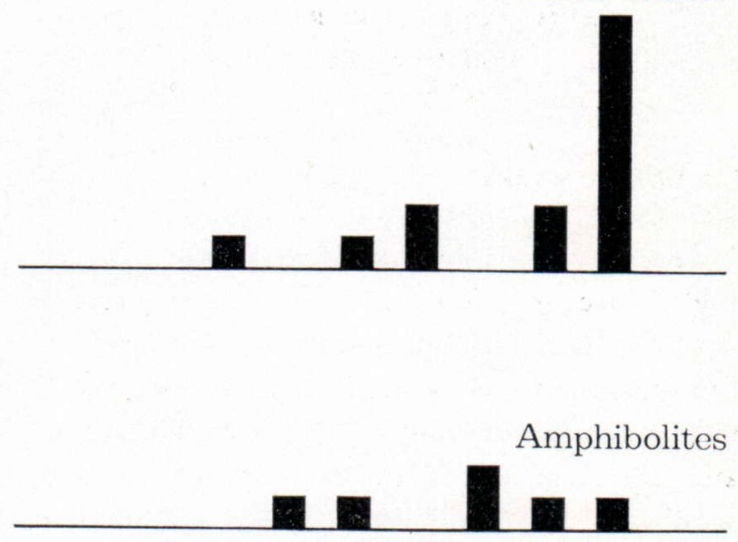

Carbonate rocks
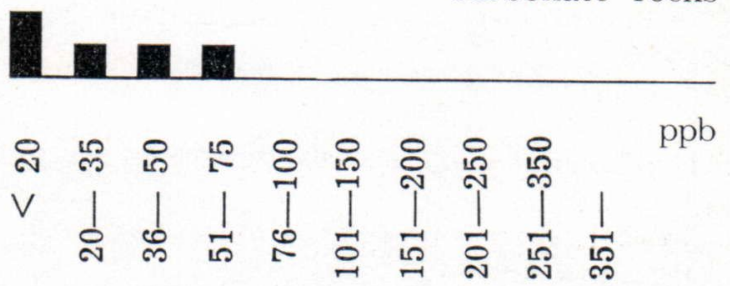

Fig. 1. Review of the selenium contents in metamorphic rock studied ( one sample) 
contents. The comparison can be made between granites with a tentative average Se content (Koljonen 1971) $25 \mathrm{ppb}$ and gneisses with a mean, according to Table 1 , of $62 \mathrm{ppb}$. The gneisses in the Lappajärvi area normally contain less selenium (Nos. 10, 11, 32). Since the meteorites contain $2000-50000 \mathrm{ppb}$ of this element (DuFresne 1960; Pelly and Lipschutz 1970; Sindeeva 1964) it can be considered possible that the selenium contents reflect the primary concentration of the meteorite, which has caused the impact, and increased the selenium content of the shock-metamorphic rocks.

\section{Summary}

The contents of selenium varies in metamorphic rocks within wider limits than in plutonic rocks. The average values presented below only indicate the general tendency of the distribution:

\begin{tabular}{|c|c|c|}
\hline & Nos. & Se ppb \\
\hline Gneiss $\ldots \ldots \ldots$ & $2-36$ & 62 \\
\hline Quartzite $\ldots \ldots \ldots$. & $37-44$ & 43 \\
\hline Conglomerate ..... & $67-70$ & 120 \\
\hline Mica schist $\ldots \ldots \ldots \ldots \ldots$ & $47-52$ & 190 \\
\hline Black schist $\ldots \ldots \ldots \ldots$ & $53-60$ & 9900 \\
\hline Amphibolite $\quad \ldots \ldots \ldots \ldots$ & $61-66$ & 240 \\
\hline Carbonate rocks $\ldots \ldots \ldots \ldots$ & $71-75$ & 25 \\
\hline Metamorphic volcanics ..... & $87-96$ & 36 \\
\hline
\end{tabular}

Selenium tends to enrich in the melanocratic rocks and also the rocks which have contained organic matter before metamorphism. This is observed as an increase in the selenium contents in amphibolites, mica schists, black schists and coals (cf. Goldschmidt 1962, 538-539). The carbonate rocks analyzed in this study are nearly monomineralic, homogeneous, crystalline limestones without any fossil remains. They may have lost the original selenium content during metamorphism. Calcium does not form any selenides and, therefore, cannot retain selenium. The selenium content of calcareous clay sediments may well be retained through metamorphism as in amphibolite and black schist (cf. Peltola 1960, 90-95), which may contain appreciable concentrations of selenium.

The selenium content in metamorphic volcanogenic rocks is unexpectedly low when compared with other silica-poor sedimentary rocks. Noteworthy is that the average content is near 54 ppb's Se which is the average of the dyke rocks: albite diabases, trachyandesites, plagioclase porphyrites and feldspar porphyrites (Koljonen 1971, 139-144) of volcanic origin.

Some of the samples of volcanogenic rocks which do not contain detectable selenium contents are from the surroundings of the town of Porvoo and especially from the island of Suur-Pellinki situated near contact to the younger Onas granite. It is, therefore, possible that volcanics have lost part of their selenium content by thermal contact metamorphism.

\footnotetext{
Acknowledgements - I thank Professor Th. G. Sahama for his interest in the present investigation, for much advice during various stages of the work, for many a fruitful discussion and for criticism of the manuscript.

The laboratory work was carried out in the laboratory of the Department of Geology and Mineralogy of the University of Helsinki. I take pleasure in thanking Professor Heikki V. Tuominen, Director of the aforesaid Department, for his liberality and kindness in allowing me freely to use his Department.
}

Mr. Olavi Y. Nurminen assisted me to handle the samples and Mr. Urpo Eklund drew the histogram.

Mr. John Nelson, Tech. Lic., checked the language of the English manuscript.

Financial support for this study was provided by grants awarded by the Finnish Cultural Foundation (Suomen Kulttuurirahasto). Finally, I would like to record my warm appreciation to my wife, Ritva, for her patience and support during the various laborious phases of my work. 
TABle 1

Selenium contents of the metamorphic rocks

\begin{tabular}{|c|c|}
\hline No. & Rock and locslity \\
\hline 1 & $\begin{array}{l}\text { Biotite gneiss, contains sulphide veinlets. Mikkeli mlk., } \\
\text { Finland (Frosterus 1900; and 1903, } 8-11 \text { ) ...... }\end{array}$ \\
\hline 2 & 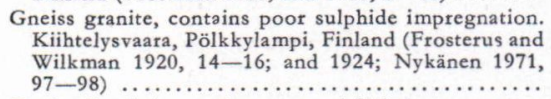 \\
\hline 3 & 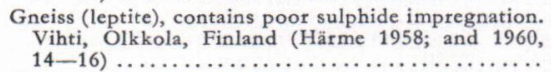 \\
\hline 4 & $\begin{array}{r}\text { Biotite gneiss. Sipoo, Skräddarby, Finland (Härme } \\
1962,113-125) \ldots \ldots \ldots \ldots \ldots \ldots \ldots \ldots \ldots \ldots\end{array}$ \\
\hline 5 & $\begin{array}{l}\text { Biotite gneiss. Vimpeli, Finland (Saksela 1934; and } \\
\quad \text { 1935, 6-7; Laitakari 1942, 11 -14) ........... }\end{array}$ \\
\hline 6 & $\begin{array}{l}\text { Quartz-feldspar schist (leptite). Ylöjärvi, Kiviniemen- } \\
\text { lahti, Finland (Simonen 1952, 10-14, 49-50; and } \\
\text { 1953b) } \ldots \ldots \ldots \ldots \ldots \ldots \ldots \ldots \ldots \ldots \ldots \ldots \ldots \ldots\end{array}$ \\
\hline 7 & $\begin{array}{l}\text { Granulite. Inari, Finland (Hackman 1905, 102; Eskola } \\
\text { 1932; Sahama 1936a, } 1-113 \text {; and b, 267-274; } \\
\text { Erämetsä 1938, 12, 49; Rankama 1946, 28-39)... }\end{array}$ \\
\hline 8 & $\begin{array}{l}\text { Cordierite-garnet gneiss. Helsinki, Oulunkylä, Finland } \\
\quad \text { (Parras 1958, 90-93; Härme 1965) .......... }\end{array}$ \\
\hline 9 & $\begin{array}{l}\text { Paragneiss. Kuopio mlk., Jynkänlahti, Finland (Wilk- } \\
\text { man 1923, 8-14; Preston 1954, 16-24) } \ldots \ldots \ldots\end{array}$ \\
\hline 10 & Gneiss (leptite). Salla, Finland (Lauerma 1967) .... \\
\hline 11 & Gneiss (leptite). Salla, Finland (Lauerma 1967) .... \\
\hline 12 & $\begin{array}{l}\text { Gneiss. Petäjävesi, Kintaus, Finland (Frosterus 1900; } \\
\text { and 1903, 8-11; Rouhunkoski } 1959,50-54) . \ldots \ldots\end{array}$ \\
\hline 13 & $\begin{array}{l}\text { Pyroxene gneiss. Espoo, Nuuksio, Finland (Sederholm } \\
\quad 1928,74-75 \text {; Parras } 1958,96-103) \ldots \ldots \ldots \ldots \ldots\end{array}$ \\
\hline 14 & $\begin{array}{l}\text { Biotite gneiss. Nastola, Finland (Lehijärvi } 1964 \text { a; and } \\
1964 \mathrm{~b}, 10-15 \text { ) } \ldots \ldots \ldots \ldots \ldots \ldots \ldots \ldots \ldots \ldots \ldots\end{array}$ \\
\hline 15 & 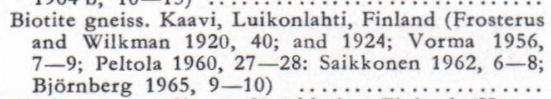 \\
\hline 16 & $\begin{array}{l}\text { Biotite gneiss. Sipoo, Skräddarby, Finland (Härme } \\
1962,113-125) \ldots \ldots \ldots \ldots \ldots \ldots \ldots \ldots \ldots \ldots \ldots \ldots \ldots \ldots\end{array}$ \\
\hline 17 & Gneiss (augen gneiss). Valàsen, Immelport, Sweden \\
\hline 18 & $\begin{array}{l}\text { Cordierite gneiss, contains pinite. Helsinki, Kivikko, } \\
\text { Finland (Parras 1958,90-93; Härme 1965) ...... }\end{array}$ \\
\hline 19 & $\begin{array}{l}\text { Pyroxene gneiss. Espoo, Nuuksio, Finland (Sederholm } \\
1928,74-75 ; \text { Simonen 1941, 130; Parras 1958, } \\
96-103) \ldots \ldots \ldots \ldots \ldots \ldots \ldots \ldots \ldots \ldots \ldots \ldots \ldots \ldots \ldots\end{array}$ \\
\hline 20 & $\begin{array}{l}\text { Biotite gneiss. Mikkeli mlk., Finland (Frosterus } 1900 \text {; } \\
\text { and } 1903,8-11) \ldots \ldots \ldots \ldots \ldots \ldots \ldots \ldots \ldots \ldots \ldots\end{array}$ \\
\hline 21 & $\begin{array}{l}\text { Biotite gneiss. Mikkeli mlk., Kalvitsa, Finland (Froste- } \\
\text { rus } 1900 \text {; and } 1903,8-11) \ldots \ldots \ldots \ldots \ldots \ldots \ldots\end{array}$ \\
\hline 22 & $\begin{array}{l}\text { Biotite-plagioclase gneiss. Heinola, Finland (Frosterus } \\
\text { 1900; and 1903, 8-11; Savolahti 1962, 48-50, } \\
\text { Table 6, Anal. 1) . . . . . . . . . . . . . . . . . }\end{array}$ \\
\hline 23 & $\begin{array}{l}\text { Gneiss. Sipoo, Skräddarby, Finland (Sederholm 1928, } \\
\quad 74-75 \text {; Härme 1962, 113-125) } \ldots \ldots \ldots \ldots \ldots \ldots\end{array}$ \\
\hline 24 & $\begin{array}{l}\text { Pyroxene gneiss. Espoo, Nuuksio, Finland (Sederholm } \\
1928,74-75 ; \text { Simonen 1941, 130; Parras 1958, } \\
96-103) \ldots \ldots \ldots \ldots \ldots \ldots \ldots \ldots \ldots \ldots \ldots \ldots \ldots \ldots \ldots\end{array}$ \\
\hline 25 & 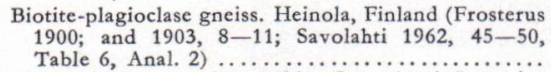 \\
\hline 26 & $\begin{array}{l}\text { Biotite gneiss, contains gaphite. Lappajärvi, Lammin- } \\
\text { kylä, Finland (Saksela 1934; and 1935, 6-7; Laita- } \\
\text { kari 1942, 38-40; Lehtinen 1970) } \ldots \ldots \ldots \ldots \ldots \text {. }\end{array}$ \\
\hline 27 & $\begin{array}{l}\text { Gneiss. Vimpeli, Finland (Saksela 1934; and 1935, } \\
6 \text {-7; Laitakari } 1942,11-14) \ldots \ldots \ldots \ldots \ldots \ldots\end{array}$ \\
\hline 28 & $\begin{array}{l}\text { Garnet-cordierite gneiss. Raisio, Finland (Parras 1958, } \\
\quad 90-93 \text {; Härme 1960,12-14) } \ldots \ldots \ldots \ldots \ldots \ldots \ldots\end{array}$ \\
\hline 29 & $\begin{array}{l}\text { Diopside gneiss. Vihti, Olkola, Finland (Parras 1958, } \\
103-107) \ldots \ldots \ldots \ldots \ldots \ldots \ldots \ldots \ldots \ldots \ldots \ldots\end{array}$ \\
\hline 30 & $\begin{array}{c}\text { Feldspar gneiss. Vantaa, Viinikkala, Finland (Härme } \\
1965) \ldots \ldots \ldots \ldots \ldots \ldots \ldots \ldots \ldots \ldots\end{array}$ \\
\hline 31 & $\begin{array}{l}\text { Cordierite-garnet gneiss (kinzigite). Helsinki, Käpylä, } \\
\text { Finland (Erämetsä 1938, 49; Parras 1958, 90-93; } \\
\text { Härme 1965, 28-32) .................. }\end{array}$ \\
\hline 32 & $\begin{array}{l}\text { Biotite gneiss. Helsinki, Käpylä, Finland (Parras 1958, } \\
90-93 \text {; Härme } 1965,11,28-32) \ldots \ldots \ldots \ldots \ldots\end{array}$ \\
\hline 33 & $\begin{array}{l}\text { Cordierite-garnet gneiss (kinzigite). Helsinki, Käpylä, } \\
\text { Finland (Erämetsä 1938, 49; Parras 1958, 90-93; } \\
\text { Härme } 1965,28-32) \ldots \ldots \ldots \ldots \ldots \ldots \ldots \ldots \ldots\end{array}$ \\
\hline
\end{tabular}

\begin{tabular}{|c|c|c|}
\hline $\begin{array}{c}\text { Selenium } \\
\text { content } \\
\mathrm{ppb}\end{array}$ & No. & Rock and locality \\
\hline 1980 & 34 & $\begin{array}{l}\text { Biotite gneiss. Siilinjärvi, Asikkala, Finland (Wilkman } \\
\text { 1933,92; Puustinen } 1968,14-15,77-79 \text {; and } 1969 \\
89-92) \ldots \ldots \ldots \ldots \ldots \ldots \ldots \ldots \ldots \ldots \ldots \ldots \ldots \ldots \ldots \ldots \ldots \ldots \ldots\end{array}$ \\
\hline & 35 & $\begin{array}{l}\text { Gneiss (fenite). Siilinjärvi, Asikkala, Finland (Wil } \\
\operatorname{man} \text { etc.) } \ldots \ldots \ldots \ldots \ldots \ldots \ldots \ldots \ldots \ldots \ldots \ldots \ldots \ldots\end{array}$ \\
\hline 320 & 36 & $\begin{array}{r}\text { Biotite gneiss. Siilinjärvi, Asikkala, Finland (Wilkm } \\
\text { etc.) } \ldots \ldots \ldots \ldots \ldots \ldots \ldots \ldots \ldots \ldots \ldots \ldots \ldots \ldots \ldots \ldots \ldots \ldots \ldots\end{array}$ \\
\hline 280 & 37 & 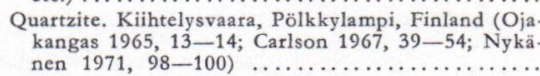 \\
\hline 250 & 38 & $\begin{array}{l}\text { Quartzite. Kiihtelysvaara, Pölkkylampi, Finland }\left(\mathrm{O}_{j a}\right. \\
\text { kangas etc.) } \ldots \ldots \ldots \ldots \ldots \ldots \ldots \ldots \ldots \ldots \ldots \ldots \ldots \ldots \ldots \ldots \ldots\end{array}$ \\
\hline 170 & 39 & $\begin{array}{l}\text { Quartzite. Kemi mlk., Akkunusjoki, Finland (Härme } \\
\text { 1949, 11-12; Enkovaara, Härme, and Väyrynen } \\
\text { 1953, 83-84; Mikkola 1960, 156-157; Kahma, } \\
\text { Siikarla, Veltheim, Vaasjoki, and Heikkinen } 1962 \\
\text { 32-33; Näykki 1964, 18-21; Ojakangas 1965, 16) }\end{array}$ \\
\hline 110 & 40 & $\begin{array}{l}\text { Quartzite. Kuopio, Neulamäki, Finland (Wilkman } \\
\text { 1923, 14-27; Preston 1954, 38-44) } \ldots \ldots \ldots \ldots \ldots\end{array}$ \\
\hline 93 & 41 & $\begin{array}{l}\text { Sericite quartzite. Eno, Pauk kajavaara, Finland (Ty } \\
1960,12-13 \text {; Ojakangas } 1965,14-15 \text {; Piirain } \\
1968,17-25) \ldots \ldots \ldots \ldots \ldots \ldots \ldots \ldots \ldots \ldots\end{array}$ \\
\hline $\begin{array}{l}92 \\
87\end{array}$ & 42 & $\begin{array}{l}\text { Quartzite, contains } 0.40 \% \text { Mn. Kiihtelysvaara, Raate } \\
\text { vaara, Finland (Ojakangas 1965, 13-14; Carlson }\end{array}$ \\
\hline 87 & 43 & $\begin{array}{l}1967,48) \ldots \ldots \ldots \ldots \ldots \ldots \\
\text { Arkose quartzite. Kontiolahti, Finland (Ojakanga } \\
1965,13-14) \ldots \ldots \ldots \ldots \ldots \ldots \ldots \ldots \ldots \ldots\end{array}$ \\
\hline 86 & 44 & $\begin{array}{l}\text { Sericite quartzite. Kiihtelysvaara, Raatevaara, Pölkky } \\
\text { lampi, Finland (Ojakangas 1965, 13-14; Carlson }\end{array}$ \\
\hline 63 & 45 & $\begin{array}{l}\text { Meta-arkose. Suodenniemi, Finland (Sederholm 1903 } \\
1913,32-34 \text {; and } 1931,43-47 \text {; Simonen } 1953 a \\
33-34) \ldots \ldots \ldots \ldots \ldots \ldots \ldots \ldots \ldots \ldots \ldots \ldots \ldots \ldots \ldots \ldots \ldots \ldots \ldots \ldots \ldots \ldots\end{array}$ \\
\hline & 46 & $\begin{array}{l}\text { Graywacke. Nokia, Finland (Simonen } 1953 a, 31-37 \\
\quad \text { Marmo } 1957,10) \ldots \ldots \ldots \ldots \ldots \ldots \ldots \ldots \ldots \ldots \ldots \ldots\end{array}$ \\
\hline 55 & 47 & $\begin{array}{l}\text { Mica schist (microfolded). Kiihtelysvaara, Heinävaara } \\
\text { Finland (Frosterus and Wilkman 1920,109-112 } \\
\text { Carlson } 1967,9-11) \ldots \ldots \ldots \ldots \ldots \ldots \ldots \ldots \ldots \ldots\end{array}$ \\
\hline 37 & 48 & $\begin{array}{l}\text { Varved mica schist. Ylöjärvi, Siivikkala, Finland } \\
\text { (Sederholm 1897, 82-93; and 1931, 10; Rankama }\end{array}$ \\
\hline 35 & & 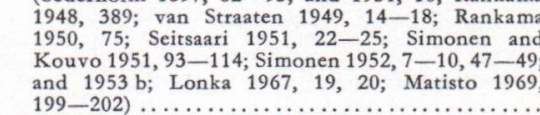 \\
\hline
\end{tabular}

Selenium content $\mathrm{ppb}$

Phyllitic mica schist. Kiihtelysvaara, Heinävaara, Finland (Frosterus and Wilkman 1920, 109-112; Carlson 1967, 9-11; Nykänen 1971, 107) ......... Mica schist. Kiihtelysvaara, Heinävaara, Finland

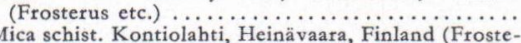
Mica schist. Kontiolahti, Heinävaara, Finland (Froste-
rus and Wilkman 1920,109-112; Lonka 1967, 32) Staurolite mica schist. Tohmajärvi, Finland (Frosterus and Wilkman 1920,110-112; Juurinen 1956, 13; Nykänen 1967; and 1971, 97, 104) Black schist. Sotkamo, Talvivaara, Finland (Wilkman 1929; and 1931, 188; Marmo 1960, 53, 58, 65; Lonka

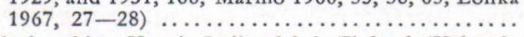

Black schist. Kaavi, Luikonlahti, Finland (Vähätalo 1953, Append. I; Peltola 1960, $27-28,78,80) \ldots$. Black schist. Kuhmoinen, Patavesi, Finland (Frosterus 1900; and 1903) 32; Laitakari 1942, 18) $\ldots \ldots \ldots \ldots \ldots \ldots \ldots \ldots \ldots$ Black schist. Pyhäselkä, Mulo, road cut, Finland (Peltola 1960, 50-57, 80; Lonka 1967, 19) .....

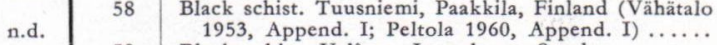

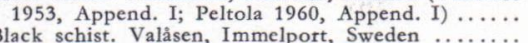
Black schist. Valåsen, Immelport, Sweden $\ldots \ldots \ldots$
Black schist. Jäppilä, Finland (Frosterus 1900$) \ldots \ldots$ n.d. $\quad \begin{array}{ll}60 & \text { Black schist. Jäppila, Finland } \\ 61 & \text { Amphibolite. Kuopio, Neulamäki, Finland (Wilkman }\end{array}$

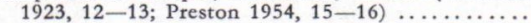

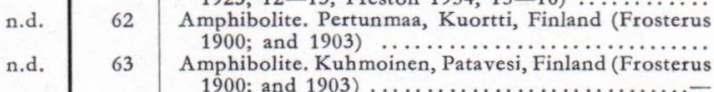

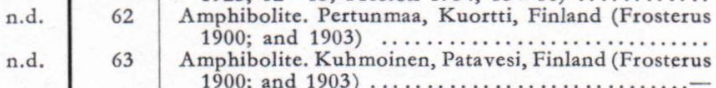

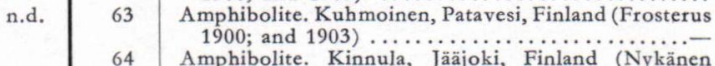
n.d. 1962; and 1963, 12-13, Table I, Anal. 1) ........ 


\begin{tabular}{|c|c|c|c|c|c|}
\hline No. & Rock and locality & $\begin{array}{c}\text { Seienium } \\
\text { content } \\
\text { pph }\end{array}$ & No. & Rock and locality & $\begin{array}{l}\text { Selenium } \\
\text { content } \\
\text { ppb }\end{array}$ \\
\hline 65 & $\begin{array}{l}\text { Amphibolite. Kuopio, Neulalahti, Finland (Wilkman } \\
1923,12-13 \text {; Preston } 1954,15-16 \text { ) } \ldots \ldots \ldots \ldots \ldots\end{array}$ & 150 & 82 & $\begin{array}{l}\text { Diopside skarn in magnetite ore. Helsinki, Laajasalo, } \\
\text { Stansvik, Finland (Tammekann 1925, 1-26; Seit- }\end{array}$ & \\
\hline 66 & 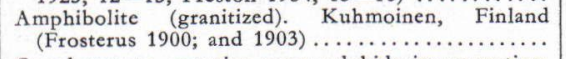 & 93 & & $\begin{array}{l}\text { saari } 1943,38-41 \text {; Aurola } 1956,11-38 \text {; Virtanen } \\
1959,36-40) \ldots \ldots \ldots \ldots \ldots \ldots \ldots \ldots \ldots \ldots \ldots \ldots \ldots \ldots\end{array}$ & n.d. \\
\hline 67 & $\begin{array}{l}\text { Conglomerate, contains poor sulphide impregnation. } \\
\text { Kiihtelysvaara, Pölkkylampi, Finland (Frosterus and } \\
\text { Wilkman 1920,148; Carlson 1967, 26_-34; Nykänen } \\
\text { 1971,98-100) …....................... }\end{array}$ & 200 & 84 & $\begin{array}{l}\text { Serpentine rock. Tuusniemi, Paakkila, Finland (Haa- } \\
\quad \text { pala 1936, 58-63; Suila 1950, 16-39; Wiik 1953, } \\
\text { 8, 10, 48; Vesasalo 1961, 11, 104) .............. } \\
\text { Serpentine rock. Tuusniemi, Paakkila, Finland (Haa- }\end{array}$ & 2810 \\
\hline 68 & $\begin{array}{l}\text { Liperi, Venehpohja, Finland (Frosterus } \\
\text { n } 1924,65-66)\end{array}$ & 160 & 85 & etc.) .................................. Find (N & 1200 \\
\hline 69 & $\begin{array}{l}\text { Conglomerate. Lavia, Harju, Finland (Sederholm 1931, } \\
11,48-58) \quad \ldots \ldots \ldots \ldots \ldots \ldots \ldots \ldots \ldots \ldots \ldots \ldots \ldots \ldots \ldots \ldots \ldots\end{array}$ & 74 & 86 & $\begin{array}{l}\text { nen 1968, 25, Anal. 1, 53-63) } \ldots \ldots \ldots \ldots \ldots \ldots \\
\text { Serpentine rock, contains amphibole. Nivala, Finland } \\
\text { (Saksela and Hackzell 1938, 73-79; Huhta 1953, }\end{array}$ & \\
\hline 70 & $\begin{array}{l}\text { Conglomerate, contains quartz pebbles. Eno, Paukka- } \\
\text { janvaara, Finland (Frosterus and Wilkman 1920, } \\
\text { 149; and 1924; Tyni 1960, 13-15) }\end{array}$ & 40 & 87 & $\begin{array}{r}\text { 21-25; Papunen 1970, } 23-25) \\
\text { Agglomerate. Ylöjärvi, Kiviniemi, Finland (Simonen } \\
\text { 1952, 17-20, Map I; and } 1953 \text { b) } \ldots \ldots \ldots \ldots \ldots \ldots\end{array}$ & 0 \\
\hline 71 & $\begin{array}{c}\text { Dolomite rock. Soanlahti, Soanjoki, USSR. (Eskola, } \\
\text { Hackman, Laitakari and Wilkman 1919, } 161-165 ; \\
\text { Hausen 1930,72-80) } \ldots \ldots \ldots \ldots \ldots \ldots \ldots \ldots \ldots\end{array}$ & 51 & 88 & 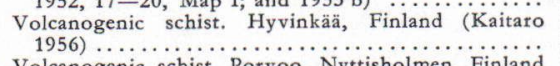 & 100 \\
\hline 72 & 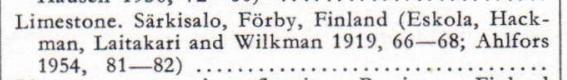 & 45 & 90 & $\begin{array}{l}\text { Volcanogenic schist. Porvoo, Nyttisholmen, Finland } \\
\text { (Sederholm 1923, 18-68; Laitala 1964) } \ldots \ldots \ldots \\
\text { Tuffite. Pernaja, Finland (Laitakari and Simonen } \\
\text { 1962; and } 1963,13-17) \ldots \ldots \ldots \ldots \ldots \ldots \ldots\end{array}$ & 66 \\
\hline 73 & 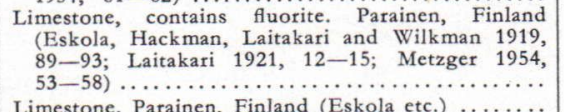 & . & $\begin{array}{l}91 \\
92\end{array}$ & 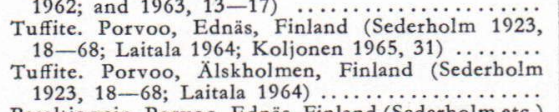 & . \\
\hline $\begin{array}{l}74 \\
75\end{array}$ & $\begin{array}{l}\text { Limestone. Parainen, Finland (Eskola ctc.) } \ldots \ldots \ldots \\
\text { Limestone. Lohja, Tytyti, Finland (Eskola, Hackman, }\end{array}$ & 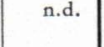 & $\begin{array}{l}93 \\
94\end{array}$ & $\begin{array}{l}\text { Basaltic vein. Porvoo, Ednäs, Finland (Sederholm etc.) } \\
\text { Uralite porphyrite (pillow lava). Porvoo, Suur-Pellinki, }\end{array}$ & \\
\hline & $\begin{array}{l}\text { Laitakari and Wilkman 1919,40-42; Kalla 1952; } \\
\text { Parras and Tavela } 1954,71-73) \ldots \ldots \ldots \ldots \ldots\end{array}$ & n.d. & 95 & $\begin{array}{l}\text { Uralite porphyrite (pillow lava). } \\
\text { Finland (Sederholm etc.) } \\
\text { Basic volcananogenic rock (u }\end{array}$ & 59 \\
\hline 76 & $\begin{array}{l}\text { Chlorite-quartz schist. Siilinjärvi, Asikkala, Finland } \\
\text { (Puustinen 1968) }\end{array}$ & & & $\begin{array}{l}\text { Hyvinkää, Kytäjä, Finland (Kaita } \\
1960,20-23) \ldots \ldots \ldots \ldots \ldots \ldots \ldots\end{array}$ & 51 \\
\hline 77 & $\begin{array}{l}\text { Talc schist. Sotkamo, Lahnaslampi, Finland (Salminen } \\
\text { 1935, 19; Wiik 1953, 51; Vesasalo 1961, 11, 103) ... }\end{array}$ & 26 & 9 & $\begin{array}{l}\text { Uralite porphyrite. Porvoo, Suur-Pel } \\
\text { (Sederholm 1923, 18-68; Laitala } 19\end{array}$ & \\
\hline 78 & $\begin{array}{r}\text { Biotite-quartz-lime schist. Siilinjärvi, A sikkala, Finland } \\
\text { (Puustinen 1968) ........................ }\end{array}$ & 145 & 97 & $\begin{array}{l}\text { Blastomylonite. Vimpeli, Koskela, River Savo, Finland } \\
\text { Shock-metamorphic tocks of the Lake Lappajärvi }\end{array}$ & 180 \\
\hline 79 & $\begin{array}{r}\text { Garnet-glaucophane schist. Tierra del Fuego, Seno } \\
\text { Martinez, Bahia Plüschow, Argentina (Kranck 1932, } \\
14,53) \ldots \ldots \ldots \ldots \ldots \ldots \ldots \ldots \ldots \ldots \ldots \ldots \ldots \ldots \ldots \ldots \ldots \ldots \ldots \ldots\end{array}$ & 31 & 98 & $\begin{array}{l}\text { area (Eskola } 1921,11-13 \text {; Saksela } 1935,5 \text {; Laitakari } \\
\text { 1942, 38-40; Lehtinen } 1970,89-93): \ldots \ldots \ldots \ldots \ldots \\
\text { Kärnäite, type II (Lehtinen, in press). Alajärvi, Kaar- }\end{array}$ & \\
\hline 80 & $\begin{array}{l}\text { Ultrabasic rock in limestone. Vihti, Olkkala, Finland } \\
\text { (Eskola, Hackman, Laitakari and Wilkman 1919, } \\
\text { 32-35; Härme 1960, Append. 2) } \ldots \ldots \ldots \ldots \ldots \ldots\end{array}$ & n.d. & & 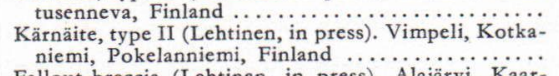 & 362 \\
\hline 81 & $\begin{array}{l}\text { Garnct skarn in magnetite ore. Helsinki, Laajasalo, } \\
\text { Stansvik, Finland (Tammekann 1925, 1-26; Sahama } \\
\text { 1936b, 273; Seitsaari 1943, 38-41; Rankama 1944, } \\
\text { 25; Aurola 1956, 13-38; Virtanen 1959, 36_40) }\end{array}$ & & $\begin{array}{l}100 \\
101\end{array}$ & $\begin{array}{l}\text { Fallout-breccia (Lehtinen, in press). Alajärvi, Kaar- } \\
\text { tusenneva, Finland ......................... } \\
\text { Ligh coloured, leached kärnäite, type II (Lehtinen, } \\
\text { in press). Alajärvi, Autioniemi, Finland } \ldots \ldots \ldots \ldots \ldots\end{array}$ & 99 \\
\hline
\end{tabular}

\section{REFERENCES}

Ahlfors, B. (1954) The limestone quarries of the firm of Karl Forström Aktiebolag. Geotek. julk. 55, $81-83$.

AurolA, E. (1956) The fibrous sepiolite deposit of Stansvik. Bull. Comm. géol. Finl. 172, 13-40.

Beath, O. A. and Gilbert, C. S. (1936) Seleniumbearing vegetation during late Cretaceous time. Science 84, No. 2187, 484-485.

Beath, O. A., Gilbert, C. S. and Eppson, H. F. (1937) Selenium in soils and vegetation associated with rocks of Permian and Triassic age. Am. J. Bot. 24, $96-101$.

Beath, O. A., Hagner, A. F. and Gilbert, C. S. (1946) Some rocks and soils of high selenium content. Wyo. Geol. Surv., Bull. 36, 1-23.

Bellini, R. (1907) Spuren von Selen auf der Vesuvlava von 1906. Zentralbl. Miner. 20, 611-612.
BJörnberG, F. (1965) Luikonlahden karsikivien petrografiaa. Unpubl. manuscr., Arch. Dep. Geol. Miner., Univ. Helsinki.

Brown, G. V. (1916) Composition of the selensulfur from Hawaii. Am. J. Sci., Ser. 4, 42, 132-134.

Brunfelt, A. O. and Steinnes, E. (1967) Determination of selenium in standard rocks by neutron activation analysis. Geochim. Cosmochim. Acta 31, 283-285.

Buryanova, E. Z. (1961) Selenium in the sedimentary rocks of Tuva. Geochemistry 7, 669-678.

Byers, H. G. and KNIGHT, H. G. (1935) Selenium in Soils. Ind. Eng. Chem., Ind. Ed. 27, 902-904.

Byers, H. G., Williams, K. T. and Lakin, H. W. (1936) Selenium in Hawaii and its probable source in the United States. Ind. Eng. Chem., Ind. Ed. 28, 821-823.

Carlson, L. (1967) Jatulimuodostumien ja niihin liittyvien metavulkaniittien stratigrafiasta Kiihtelysvaa- 
ran pitäjän lounaisosissa. Unpubl. manuscr., Arch. Dep. Geol. Miner., Univ. Helsinki.

Chentzov, I. G. (1963) Selenium in Paleogene deposits of Central Asia. Mineral. Abstr. 15, 189-190.

Clarke, F. W. (1924) The data of geochemistry. U. S. Geol. Surv., Bull. 770, 19, 186, 273.

Coleman, R. G. (1956) The occurrence of selenium in sulfides from sedimentary rocks of the western United States. Econ. Geol. 51, 112.

Coleman, R. G. and Delevaux, M. (1957) Occurrence of selenium in sulfides from some sedimentary rocks of the western United States., Econ. Geol. 52, 499527.

Davidson, D. F. and Gulbrandsen, R. A. (1957) Selenium in the Phosphoria formation in Idaho, Wyoming, Utah, and Montana. Bull. Geol. Soc. Am. 68, 1714.

Davidson, D. F. and Powers, H. A. (1959) Selenium content of some volcanic rocks from western United States and Hawaiian Islands. U. S. Geol. Surv., Bull. 1084-C, 69-81.

DuFresne, A. (1960) Selenium and tellurium in meteorites. Geochim. Cosmochim. Acta 20, 141-148.

Enkovaara, A., Härme, M. and Väyrynen, H. (1953) Kivilajikartan selitys C5-B5, Oulu-Tornio. English summary. General Geological map of Finland, $1: 400$ 000. Geologinen tutkimuslaitos, Otaniemi

ERÄMETSÄ, O. (1938) Über die Verbreitung des Indiums in finnischen Mineralen und über seine Trennung von anderen Metallen. Ann. Acad. Sci. Fenn., Ser. A, III, LI : 1 .

EsкоцA, P. (1921) On volcanic necks in Lake Jänisjärvi in eastern Finland. Bull. Comm. géol. Finl. 55.

- (1932) On the origin of granitic magmas. Min. Petr. Mitt. 42, 455-481.

Eskola, P., Hackman, V., Laitakari, A. and Wilkman, W. W. (1919) Suomen kalkkikivi. Summary: Limestones in Finland. Geotek. julk. 21.

Fleming, G. A. and Walsh, T. (1957) Selenium occurrence in certain Irish soils and its toxic effects on animals. R. Ir. Acad. Proc. 58, Sec. B, 151-166.

Frosterus, B. (1900) General geological map of Finland, 1 : 400 000, Sheet C2, Mikkeli. Geologinen tutkimuslaitos, Otaniemi.

- (1903) Vuorilajikartan selitys C2, Mikkeli. General geological map of Finland, 1:400 000. Geologinen tutkimuslaitos, Otaniemi.

Frosterus, B. and Wilkman, W. W. (1920) Vuorilajikartan selitys D3, Joensuu. General geological map of Finland, 1:400 000. Geologinen tutkimuslaitos, Otaniemi.

- (1924) General geological map of Finland, $1: 400$ 000, Sheet D3, Joensuu. Geologinen tutkimuslaitos, Otaniemi.
Gibson, F. H. and Selvig, W. A. (1944) Rare and uncommon chemical elements in coal. U. S. Bur. Mines, Tech. Pap. 669.

Goldschmidt, V. M. (1962) Geochemistry. Clarendon Press, Oxford.

GreEN, J. (1959) Geochemical table of the elements for 1959. Bull. Geol. Soc. Am. 70, 1127-1184.

HaApala, P. (1936) On serpentine rocks in northern Karelia. Bull. Comm. géol. Finl. 114.

Haскмan, V. (1905) Die chemische Beschaffenheit von Eruptivgesteinen Finlands und der Halbinsel Kola im Lichte des neuen amerikanischen Systemes. Bull. Comm. géol. Finl. 15.

Hausen, H. (1930) Geologie des Soanlahti-Gebietes im südlichen Karelien. Ein Beitrag zur Kenntnis der Stratigraphie und tektonischen Verhältnisse der Jatulformation. Bull. Comm. géol. Finl. 90.

Hunma, A. (1970a) Short description of the geology of the Outokumpu district. Bull Geol. Soc. Finl. 42, 59-66.

- (1970b) Outokummun alueen kvartsiiteista, karsista ja karbonaattikivistä. Unpubl. thesis, Arch. Dep. Geol. Miner., Univ. Helsinki.

Huнta, J. (1953) Nivalan pitäjän malmiesiintymistä. Unpubl. manuscr., Arch. Dep. Geol. Miner., Univ. Helsinki.

HÄRME, M. (1949) On the stratigraphical and structural geology of the Kemi area, northern Finland. Bull. Comm. géol. Finl. 147.

- (1958) General geological map of Finland, 1: 400 000, Sheet B1, Turku. Geologinen tutkimuslaitos, Otaniemi.

- (1960) Kivilajikartan selitys B1, Turku. English summary. General geological map of Finland, 1 : 400 000. Geologinen tutkimuslaitos, Otaniemi.

- (1962) An example of anatexis. Bull. Comm. gćol. Finl. 204, 113-125.

- (1965) On the potassium migmatites of southern Finland. Bull. Comm. géol. Finl. 219.

JuUrinen, A. (1956) Composition and properties of staurolite. Ann. Acad. Sci. Fenn., Ser. A, III, 47.

Kahma, A., Sirkarla, T., Veltheim, V., Vaasjoki, O. and Heikninen, A. (1962) On the prospecting and the geology of the Kemi chromite deposit, Finland. Bull. Comm. géol. Finl. 194.

KaItaro, S. (1956) Geological map of Finland, 1 : 100 000, Sheet 2044, Riihimäki. Geologinen tutkimuslaitos, Otaniemi.

KALLA, J. (1952) Tytyrin kalkkikiviesiintymä Lohjalla. Unpubl. manuscr., Arch. Dep. Geol. Miner., Univ. Helsinki.

KeYs, W. S. and White, R. L. (1956) Investigation of the Temple Mountain collapse and associated fea- 
tures, San Rafael Swell, Emery County, Utah. U. S. Geol. Surv., Prof. Pap. 300, 285-298.

Koljonen, T. (1965) Seleenin esiintymisestä suomalaisissa kivissä. Unpubl. manuscr., Arch. Dep. Geol. Miner., Univ. Helsinki.

- (1971) Selenium in certain igneous rocks. Unpubl. thesis, Arch. Dep. Geol. Miner., Univ. Helsinki.

- (1973) Selenium in certain igneous rocks. Bull. Geol. Soc. Finl. 45, 9-22.

Kranck, E. H. (1932) Geological investigations in the Cordillera of Tierra del Fuego. Acta Geogr. 4: 2.

Krauskopf, K. B. (1955) Sedimentary deposits of rare metals. Econ. Geol., 50th Anniv. Vol., 411-463.

Laitakari, A. (1921) Úber die Petrographie und Mineralogie der Kalksteinlagerstätten von Parainen (Pargas). Bull. Comm. géol. Finl. 54.

- (1942) Kivilajikartan selitys B3, Vaasa. Zusammenfassung. General geological map of Finland, 1 : 400000 . Geologinen tutkimuslaitos, Otaniemi.

LaItakari, I. and Simonen, A. (1962) Geological map of Finland, 1 : 100 000, Shcet 3022, Lapinjärvi. Geologinen tutkimuslaitos, Otaniemi.

- (1963) Kallioperäkartan selitys 3022, Lapinjärvi. English summary: Explanation to the map of rocks. Geological map of Finland, 1: 100 000. Geologinen tutkimuslaitos, Otaniemi.

Laitala, M. (1964) Geological map of Finland, 1 : 100 000, Sheet 3021, Porvoo. Geologinen tutkimuslaitos, Otaniemi.

- (1965) Geological map of Finland, $1: 100$ 000, Sheet 3012, Pellinki. Geologinen tutkimuslaitos, Otaniemi.

Lakin, H. W., Williams, K. T. and Byers, H. G. (1938) »Nontoxic» seleniferous soils. Ind. Eng. Chem., Ind. Ed. 30, 599-600.

Lauerma, R. (1967) Geological map of Finland, $1: 100000$, Sheet 4621-4623, Salla. Geologinen tutkimuslaitos, Otaniemi.

Lenrjärvi, M. (1964a) Geological map of Finland, 1 : 100 000, Sheet 3111, Lahti. Geologinen tutkimuslaitos, Otaniemi.

- (1964b) Kallioperakartan sclitys 3111, Lahti. English summary: Explanation to the map of rocks. Geological map of Finland, $1: 100000$. Geologinen tutkimuslaitos, Otaniemi.

Lehtinen, M. (1970) New evidence for an impact origin of Lake Lappajärvi, western Finland. A preliminary report. Bull. Geol. Soc. Finl. 42, 89-93.

LonkA, A. (1967) Trace-elements in the Finnish Precambrian phyllites as indicators of salinity at the time of sedimentation. Bull. Comm. géol. Finl. 228.

Marmo, V. (1957) Geology of the Nokia region, Southwest Finland. Bull. Comm. géol. Finl. 176.

- (1960) On the sulphides and sulphide-graphite schists of Finland, with an especial reference to the sulphide- graphite schists of Central Pohjanmaa. Bull. Comm. géol. Finl. 190.

Matisto, A. (1969) On the microfossils of Corycium enigmaticum. Bull. Geol. Soc. Finl. 41, 199-202.

Meixner, H. (1954) Neue Mineralfunde in der österreichischen Ostalpen XIII. Carinthia II 64, 18-29.

Metzger, A. A. T. (1954) The deposit of crystalline limestone of Pargas - Parainen. Geotek. julk. 55, 53-58.

Miknola, T. (1960) Sedimentation of quartzite in the Kemi area, North Finland. Int. Geol. Congr. XXI, Part IX, 154-161.

Miller, J. T. (1937) Selenium in plants in relation to its occurrence in soils. J. Agric. Res. 55, 59-68.

Miller, J. T. and Brown, I. C. (1938) Observations regarding soils of northern and central Mexico. Soil Sci. $46,427-450$.

Moxon, A. L., Olson, O. E., Searight, V. V. and SANDALS, K. M. (1938) The stratigraphic distribution of selenium in the Cretaceous formations of South Dakota and the selenium content of some associated vegetation. Am. J. Bot. 25, 794-809.

Nuutulainen, J. (1968) On the geology of the Misi iton ore province, northern Finland. Ann. Acad. Sci. Fenn., Ser A, III, 96.

NyKäNEN, O. (1962) Geological map of Finland, 1 : 100 000, Sheet 2334, Kinnula. Geologinen tutkimuslaitos, Otaniemi.

- (1963) Kallioperäkartan selitys 2334, Kinnula. English summary: Explanation to the map of rocks. Geological map of Finland, 1:100 000. Geologinen tutkimuslaitos, Otaniemi.

- (1967) Geological map of Finland, 1 : 100 000, Sheet 4232-4234, Tohmajärvi. Geologinen tutkimuslaitos, Otaniemi.

- (1971) On the Karelides in the Tohmajärvi area, eastern Finland. Bull. Geol. Soc. Finl. 43, 93-108.

NäҮккI, О. (1964) Kemin - Kivaloiden emäksinen kompleksi. Vihreäkivet ja kerroksellinen magmakivisarja. Unpubl. thesis, Arch. Dep. Geol. Miner., Univ. Helsinki.

OJakangas, R. W. (1965) Petrography and sedimentation of the Precambrian jatulian quartzites of Finland. Bull. Comm. géol. Finl. 214.

OKSANEN, H. (1965) Studies on nutrional muscular degeneration (NMD) in ruminants. Acta Vet. Scand., Suppl. 2.

Olson, O. E., Jornlin, D. F. and Moxon, A. L. (1942) Field studies on methods for determining availability of selenium to plants. Soil Sci. 53, 365-368.

Olson, O. E., Whitehead, E. I. and Moxon, A. L. (1942) Occurrence of soluble selenium in soils and its availability to plants. Soil Sci. $54,47-53$. 
Papunen, H. (1970) Sulfide mineralogy of the Kotalahti and Hitura nickel-copper ores, Finland. Ann. Acad. Sci. Fenn., Ser. A, III, 109.

PARRAS, K. (1958) On the charnockites in the light of a highly metamorphic rock complex in southwestern Finland. Bull. Comm. géol. Finl. 181.

Parras, K. and Tavela, M. (1954) The limestone deposits in Lohja. Geotek. julk. 55, 69-74.

Pelly, I. Z. and Lipschutz, M. E. (1970) Selenium. Elemental abundances in meteoritic matter. (Ed. Mason, B.) Prepr.

Peltola, E. (1960) On the black schists in the Outokumpu region in eastern Finland. Bull. Comm. géol. Finl. 192.

Pirrainen, T. (1968) Die Petrologie und die Uranlagerstätten des Koli-Kaltimogebiets im finnischen Nordkarelien. Bull. Comm. géol. Finl. 237.

Preston, J. (1954) The Geology of the pre-Cambrian rocks of the Kuopio district. Ann. Acad. Sci. Fenn., Ser. A, III, 40.

PuUstinen, K. (1968) Siilinjärven apatiittimuodostuman geologiasta. Unpubl. thesis, Arch. Dep. Geol. Miner., Univ. Helsinki.

- (1969) The carbonatite of Siilinjärvi in the Precambrian of eastern Finland. I.ithos 3, 89-92.

Quercigh, E. (1925) Sul solfoselenio di Vulcano (Isole Eclie). R. Accad. Sci. Fis. Mat. Napoli 31, 65-69 (Chem. Abstr. 20, 2804).

RANKAMA, K. (1944) On the geochemistry of tantalum. Bull. Comm. géol. Finl. 133.

- (1946) On the geochemical differentiation in the earth's crust. Bull. Comm. géol. Finl. 137.

- (1948) New evidence of the origin of pre-Cambrian carbon. Bull. Geol. Soc. Am. 59, 389-416.

- (1950) Corycium resuscitatum: A discussion. J. Geol. $58,75-79$.

Robinson, W. O. (1933) Determination of selenium in wheat and soils. J. Assoc. Off. Agric. Chem. 16, $423-424$.

Rouhunkoski, P. (1959) Petäjäveden alueen geologiaa. Unpubl. thesis, Arch. Dep. Geol. Miner., Univ. Helsinki.

Sahama, Th. G. (1936a) Die Regelung von Quarz und Glimmer in den Gesteinen der finnisch-lappländischen Granulitformation. Bull. Comm. géol. Finnl. 113.

- (1936b) Akzessorische Elemente in den Granuliten von Finnisch-Lappland. Bull. Comm. géol. Finl. $115,267-274$.

SAIKKONEN, R. (1962) Luikonlahden serpentiinikivien petrografiaa. Unpubl. manuscr., Arch. Dep. Geol. Miner., Univ. Helsinki.

SAKSELA, M. (1934) General geological map of Finland,
$1: 400$ 000, Sheet B3, Vaasa. Geologinen tutkimuslaitos, Otaniemi.

- (1935) UUber den geologischen Bau Süd-Ostbothniens. Bull. Comm. géol. Finl. 110.

Saksela, M. and Hackzell, E. (1938) Tutkimuksia Nivalan malmista ja sen rikastamismahdollisuuksista. Suom. Kemistil., A XI: 7-8.

SALMINEN, A. (1935) On the weathering of rocks and the composition of clays. Ann. Acad. Sci. Fenn., Ser A, XLIV: 6.

Savolahti, A. (1962) The rapakivi problem and the rules of idiomorphism in minerals. Bull. Comm. géol. Finl. 204, 33-111.

Searight, W. V., Moxon, A. L., Hilmoe, R. J. and WhiteheAd, E. I. (1946) Occurrence of selenium in Pleistocene deposits and their derivatives in South Dakota. Soil Sci. 61, 455--463.

Sederholm, J. J. (1897) Über eine archäische Sedimentformation im südwestlichen Finland und ihre Bedeutung für die Erklärung der Entstehungsweise des Grundgebirges. Bull. Comm. géol. Finl. 6 .

- (1903) General geological map of Finland, 1 : 400000 , Sheet B2, Tampere. Geologinen tutkimuslaitos, Otaniemi.

- (1913) Vuorilajikartan selitys B2, Tampere. General geological map of Finland, 1:400 000. Geologinen tutkimuslaitos, Otaniemi.

- (1923) On migmatites and associated pre-Cambrian rocks of southwestern Finland. Part 1. The Pellinge region. Bull. Comm. géol. Finl. 58.

- (1928) On orbicular granites, spotted and nodular granites etc. and on the rapakivi texture. Bull. Comm. géol. Finl. 83.

- (1931) On the sub-Bothnian unconformity and on Archaean rocks formed by secular weathering. Bull. Comm. géol. Finl. 95.

SertsaAri, J. (1943) Degerön ja Santahaminan kallioperä. Unpubl. manuscr., Arch. Dep. Geol. Miner., Univ. Helsinki.

- (1951) The schist belt northeast of Tampere in Finland. Bull. Comm. géol. Finl. 153.

SIMONEN, A. (1941) Orbicular rocks in Kemijärvi and Esbo. Bull. Comm. géol. Finl. 126, 107-140.

- (1952) Kallioperäkartan selitys 2124, ViljakkalaTeisko. English summary: Explanation to the map of rocks. Geological map of Finland, 1: 100000. Geologinen tutkimuslaitos, Otaniemi.

- (1953a) Stratigraphy and sedimentation of the Svecofennidic, early Archean supracrustal rocks in southwestern Finland. Bull. Comm. géol. Finl. 160.

- (1953b) Geological map of Finland, 1:100000, Sheet 2124, Viljakkala-Teisko. Geologinen tutkimuslaitos, Otaniemi. 
Simonen, A. and Kouvo, O. (1951) Archean varved schists north of Tampere in Finland. Bull. Comm. géol. Finl. 154, 93-114.

Sindeeva, N. D. (1964) Mineralogy and types of deposits of selenium and tellurium. Interscience Publishers, New York-London-Sydney.

van Straaten, L. M. J. U. (1949) Occurrence in Finland of structures due to subaqueous sliding of sediments. Bull. Comm. géol. Finl. 144, 9-18.

Suila, M. (1950) Paakkilan asbestialueen ja asbestiesiintymien geologiasta. Unpubl. manuscr., Arch. Dep. Geol. Miner., Univ. Helsinki.

Tammekann, A. (1925) Über die Petrographie des Grubenfeldes von Sillböle bei Helsinki (Helsingfors). Fennia 45: 10 .

Trelease, S. F. and Beath, O. A. (1949) Selenium, its geological occurrence and its biological effects in relation to botany, chemistry, agriculture, nutrition, and medicine. Published by the authors, New York.

TyNI, M. (1960) Paukkajanvaaran uraanikaivosalueen kivilajeista. Unpubl. manuscr., Arch. Dep. Geol. Miner., Univ. Helsinki.

Vesasalo, A. (1961) Suomen talkki- ja vuolukiviesiintymät. Unpubl. thesis, Arch. Dep. Geol. Miner., Univ. Helsinki.

Virtanen, P. (1959) Hevossalmen-Stansvikin alueen kallioperä Laajasalossa. Unpubl, manuscr., Arch. Dep. Geol. Miner., Univ. Helsinki.

VORMA, A. (1956) Luikonlahden malmin ja metamorfisten kivien petrografiaa. Unpubl. manuscr., Arch. Dep. Geol. Miner., Univ. Helsinki.
VÄhätalo, V. O. (1953) On the geology of the Outokumpu ore deposit in Finland. Bull. Comm. géol. Finl. 164.

WIIK, H. B. (1953) Composition and origin of soapstone. Bull. Comm. géol. Finl. 165.

Wilkman, W. W. (1923) Kuopion seudun kivilajit. Geotek. julk. 36.

- (1929) General geological map of Finland, 1 : 400 000, Sheet C4, Kajaani. Geologinen tutkimuslaitos, Otaniemi.

- (1931) Beskrivning till bergartskartan C4, Kajaani. Résumé en francais. General geological map of Finland, $1: 400000$. Geologinen tutkimuslaitos, Otaniemi.

- (1933) Über zwei Syenitvorkommen in Nilsiä, nördl. von Kuopio. Bull. Comm. géol. Finl. 101, 91-97.

Williams, K. T. and Byers, H. G. (1935) Occurrence of selenium in the Colorado River and some of its tributaries. Ind. Eng. Chem., Anal. Ed. 7, 431-432.

- (1936) Selenium compounds in soils. Ind. Eng. Chem., Ind. Ed. 28, 912-914.

Zambonini, F. and Coniglio, L. (1925) The presence of soluble compounds of selenium and tellurium as products of the activity of Vesuvius. Ann. Oss. Vesuviano (3) 2, 3-6.

Zies, E. G. (1929) The Valley of Ten Thousand Smokes. Natl. Geogr. Soc. Contrib., Tech. Pap. 1: $4,1-79$.

Manuscript received, November 8, 1972. 